\title{
Comparison of Mortality in Fournier's Gangrene with the Two Scoring Systems
}

\author{
Seracettin Egin, Sedat Kamali, Semih Hot, Berk Gökçek and Metin Yesiltas \\ Department of Surgery, University of Health Sciences, Okmeydani Education and Research Hospital, Istanbul, Turkey
}

\begin{abstract}
Objective: To determine the factors associated with mortality in Fournier's gangrene (FG) toward informing the development of effective treatment strategies.

Study Design: A descriptive study.

Place and Duration of Study: Department of Surgery, University of Health Sciences, Okmeydani Education and Research Hospital, Istanbul, Turkey, from February 2012 to September 2017.

Methodology: Informations of 30 patients treated for FG were examined retrospectively. The deceased patients (Group 1, $n=8$ ) were analysed separately from the living ones (Group 2, $n=22$ ). Informations in this analysis contained gender, age score, dissemination score, Uludag Fournier's Gangrene Severity Index (UFGSI) and Fournier's Gangrene Severity Index (FGSI) scores, serum levels of urea, infection source, the presence of diabetes, obesity, and other comorbidities, the presence of stoma for diversion, duration of the vacuum-assisted closure treatment, hospitalisation time, intensive care period, and species of bacteria isolated.

Results: The overall mortality rate was detected as $26 \%$. A significant difference between the two groups concerning age score of UFGSI parameters was found. The dissemination score of the infection, which is one of the UFGSI parameters, was significantly higher in Group 1 than in Group 2. According to UFGSI and FGSI scores, the scores of group 1 patients were significantly higher. To determine the incidence of mortality, the UFGSI and FGSI had $87.5 \%$ sensitivity and had $96 \%$ and $91 \%$ specificity, respectively. The cut-off values for UFGSI and FGSI were 10 and seven, respectively.

Conclusion: Based on the findings described in this study, age and dissemination scores from the UFGSI can be used to predict patient outcome. Patients with a UFGSI score greater than 10 have a higher mortality rate.
\end{abstract}

Key Words: Fournier's gangrene, Fournier's gangrene severity index, Uludag Fournier's gangrene severity index.

How to cite this article: Egin S, Kamali S, Hot S, Gökçek B, Yesiltas M. Comparison of mortality in Fournier's gangrene with the two scoring systems. J Coll Physicians Surg Pak 2020; 30(1):67-72.

\section{INTRODUCTIONF}

Fournier's gangrene $(F G)$ is necrotizing fasciitis of the perianal and genital regions, caused by interdependent polymicrobial infections. Identifying the symptoms early is critical as delay in diagnosis and treatment can be fatal. The clinical presentation of this disease is highly variable, ranging from early, localised skin hardening, redness, and swelling to more obvious dermal indications during the later stages including up to sepsis, which is a systemic finding. FG usually begins with perianal or perineal pain. ${ }^{1}$ Scrotal swelling, deep local erythema, hyperemia, pruritus, fever, and nonspecific abdominal pain are other common symptoms. Minimal skin lesions in the early phase of FG may lead to the disease being overlooked because a definitive diagnosis is difficult. The rapid and aggressive nature, symptoms, and outcomes of this disease can sometimes manifest

Correspondence to: Dr. Seracettin Egin, Department of

Surgery, University of Health Sciences, Okmeydani Education and Research Hospital, Istanbul, Turkey

E-mail: seracettin_egin@hotmail.com

Received: March 05, 2019; Revised: May 18, 2019;

Accepted: June 19, 2019 themselves in apparent findings such as deep tanning, cyanosis, foul-smelling flow, and even necrosis.1-3 The repulsive faecaloid odour is another characteristic of the disease. Black dermal necrosis prevails with the development of gangrene. Reduction or even loss of pain with gangrene formation is typical. 4

The disease was described by Jean Alfred Fournier, dermatologist and venereologist in Paris, in 1883.2-4 Present, FG affects every age group but its incidence increases after 50 years of age. ${ }^{4}$ Interestingly, FG is closely related to low socioeconomic status and it is more prevalent in low-income societies. ${ }^{2}$ The infection is most frequently of colorectal origin $(30-50 \%)$ in terms of etiology, followed by the urological (20-40\%) and dermal $(20 \%){ }^{3}$ It is frequently associated with accompanying systemic disorders, independent of the primary effect. For example, diabetes mellitus (DM) has been detected in $20-70 \%$ of FG cases. 1,3 Chronic alcoholism is the second most common cause (25-50\%). ${ }^{1,3}$ The underlying element shared by all of these associated risk factors is the impaired of immune resistance due to reduced cellular immunity. ${ }^{2}$

The main bases of treatment are contained hemodynamic stabilisation, parenteral appropriate antibiotics, 
and urgently aggressive surgical debridement.2-4 However, the basis of this triple combined treatment is early surgical debridement.

Mortality rates from FG were fallen below $40 \%$ in 2006.3 In 2000 , the overall mortality rate was found to be $16 \%$ in the study conducted by Eke.2 Most disease-related causes of mortality involve sepsis, renal insufficiency, coagulopathy , diabetic ketoacidosis-induced coma, and multiple organ failure. Advanced age, widespread disease prevalence, delayed treatment, septic shock upon hospital admission, positive blood culture, increased urea level, an anorectal infection source, diabetes, and immunosuppressive disorders are all prognostic factors that negatively influence patient survival.

Therefore, the objective of the study was to determine the factors responsible for mortality in patients with FG and develop effective treatment practices. We hypothesised that mortality expectancy increased in patients with high numbers of intensive care days and comorbidities and also in patients with high FGSI and UFGSI scores.

\section{METHODOLOGY}

Ethics approval was obtained from the Okmeydani Education and Research Hospital Ethics Board. Information recorded of 30 patients treated for FG between February 2012 and September 2017 at Okmeydani Education and Research Hospital General Surgery Clinic were investigated retrospectively. The deceased patients (Group $1, \mathrm{n}=8$ ) were analysed separately from the living ones (Group $2, n=22$ ). Data included for analysis described sex, age, infection extent, UFGSI and FGSI scores, serum levels of urea, the source of the infection, the presence of diabetes, obesity, and other comorbidities, the presence of stoma for diversion, duration of vacuum-assisted closure (VAC) treatment in days, hospitalisation time (HT) in days, intensive care period (ICP) in days, and isolated bacterial species.

The study includes a total of 30 patients over 41 years treated for FG. The patients who received VAC treatment only after aggressive surgical debridements were included in the study. Patients who did not undergo VAC treatment after surgical debridements were not evaluated. These patients were affected in narrow localised regions. These were the patients whose dermis and subdermal layer were closed with primary suture after one or two surgical debridements.

Despite the lack of a reliable tool to predict FG severity, some scoring systems have been used with moderate levels of success. A reliable scoring system should contain clear and effective data for the patient. In addition, it should be able to faithfully identify high morbidity and mortality rates. ${ }^{5}$ Laor et al. described the FGSI by adapting the acute physiological and chronic health assessment (APACHE II) score relating to the prognosis of $\mathrm{FG} .6$ They have shown that the FGSI score can be estimated accurately by $75 \%$ for mortality and $78 \%$ for survival. The FGSI score, which arises considerable interest in the literature, is a valid and effective score that widely used in many papers to determine the outcomes of FG. A new scoring system was created by Yilmazlar et al. from combining FGSI with age and dissemination score. 7 The most important feature of this scoring system, called the Uludag FGSI score, is the grading of the disease's dissemination. Czymek et al. described that the female sex is an important factor that confers poor prognosis. ${ }^{8}$ They showed that FG occurs more often in the retroperitoneal and abdominal regions of women.

Our surgical team started the operations not only for debridement or the drainage of an abscess but also for a large surgical procedure. Surgical debridements were performed widely up to live tissues thoroughly perfused. A series of re-explorations were performed every 24-48 hours for necessary debridement. The aim of aggressive debridement is to eliminate all necrotic regions, stop the dissemination of the infection, and reduce systemic toxicity. 2 The reoperations were continued until the infection was thoroughly controlled. The protection of debridement region from becoming dirty with faeces and urine may be mandatory with faecal or urinary diversion. While no consensus for colostomy can be obtained, it is recommended performing if there are sphincter involvement or large wounds in the perineum. ${ }^{1}$ The decision to open the colostomy was performed in the second debridement session where the sphincters could be better evaluated and the inflammation had highly decreased.

Because of aggressive surgical debridement, the common outcome is extensive tissue defects. For this reason, wound care in FG is an important part of the treatment. The VAC procedure, which has popularity in recent years, has made significant contributions to this period of the disease, which is extremely distressing with minimal skin defects by accelerating wound healing. ${ }^{9}$ VAC treatment was performed to all our patients after the surgical debridement procedures had been finished. VAC procedures were repeated in three- to four-day intervals. The final step in the management of $F G$ is to close the large wound after the creation of the granulation tissue is provided by VAC treatment. The wounds with small-defects simply heal spontaneously as a secondary. Sometimes, the closure of these wounds may be possible with delayed primary suture or $\mathrm{V}-\mathrm{Y}$ local advancement flaps. However, the closure with splitthickness skin grafts is commonly carried out and preferred method in case of widespread wounds. We remedied all the patients in Group 2 using three different methods, chosen according to the width of the wound (Table I). 
Table I: Characteristics of the groups and related factors according to mortality rate.

\begin{tabular}{|c|c|c|c|}
\hline & $\begin{array}{l}\text { Group } 1 \\
\text { (Exitus) }\end{array}$ & $\begin{array}{c}\text { Group } 2 \\
\text { (Alive) }\end{array}$ & $\mathrm{p}$ \\
\hline \multicolumn{4}{|l|}{ Sex } \\
\hline Female & $6(\% 42.8)$ & $8(\% 57.2)$ & 0.065 \\
\hline Male & $2(\% 12.5)$ & $14(\% 87.5)$ & \\
\hline \multicolumn{4}{|l|}{ Infection source } \\
\hline Urogenital & $6(\% 35.3)$ & $11(\% 64.7)$ & 0.22 \\
\hline Anorectal & $2(\% 15.4)$ & $11(\% 84.6)$ & \\
\hline \multicolumn{4}{|l|}{ Diabetes Mellitus } \\
\hline Yes & $7(\% 25)$ & $21(\% 75)$ & 0.44 \\
\hline No & $1(\% 50)$ & $1(\% 50)$ & \\
\hline \multicolumn{4}{|l|}{ Comorbidity } \\
\hline Yes & $8(\% 40)$ & $12(\% 60)$ & 0.02 \\
\hline No & $0(\% 0)$ & $10(\% 100)$ & \\
\hline \multicolumn{4}{|l|}{ Obesity } \\
\hline Yes & $3(\% 27.3)$ & $8(\% 72.7)$ & 0.95 \\
\hline No & $5(\% 26.3)$ & $14(\% 73.7)$ & \\
\hline \multicolumn{4}{|l|}{ Isolated bacteria type } \\
\hline None & $2(\% 22.2)$ & $7(\% 77.8)$ & 0.92 \\
\hline E.Coli & $3(\% 30)$ & $7(\% 70)$ & \\
\hline Others & $3(\% 27.3)$ & $8(\% 72.7)$ & \\
\hline \multicolumn{4}{|l|}{ Definitive closure type } \\
\hline None (Exitus) & $8(\% 100)$ & $0(\% 0)$ & 0.00 \\
\hline Primary closure & $0(\% 0)$ & $5(\% 100)$ & \\
\hline Split thickness skin graft & $0(\% 0)$ & $12(\% 100)$ & \\
\hline V-Y flap & $0(\% 0)$ & $5(\% 100)$ & \\
\hline \multicolumn{4}{|l|}{ Stoma } \\
\hline Yes & $0(\% 0)$ & $5(\% 100)$ & 0.14 \\
\hline No & $8(\% 32)$ & $17(\% 68)$ & \\
\hline
\end{tabular}

Table II: The means and statistical significance of the groups' characteristics.

\begin{tabular}{l|c|c|c}
\hline & $\begin{array}{c}\text { Group1 } \\
\text { (Exitus) }\end{array}$ & $\begin{array}{c}\text { Group 2 } \\
\text { (Alive) }\end{array}$ & $p$ \\
\hline Age (years) & $68.5 \pm 12.08^{*}$ & $55.13 \pm 9.17^{*}$ & 0.017 \\
Temperature $\left({ }^{\circ} \mathrm{C}\right)$ & $38.55(1.18)$ & $37.2(0.42)$ & 0.031 \\
Heart rate $(/ \mathrm{min})$ & $103(29)$ & $90(9)$ & 0.035 \\
Respiratory rate (/min) & $27(9.5)$ & $23(2)$ & 0.040 \\
Serum potassium (milimol/L) & $3.3(0.59)$ & $3.9(1.15)$ & 0.016 \\
Serum sodium (mmol/L) & $137.5(7.25)$ & $138.5(5.25)$ & 0.534 \\
Serum creatinine (mg/100 ml) & $1.11(0.95)$ & $1.15(0.74)$ & 0.420 \\
Haematocrit (\%) & $30.42 \pm 4.21^{*}$ & $36.08 \pm 6.31^{*}$ & 0.027 \\
White blood count (X1000/mm3) & $20.96 \pm 4.97^{*}$ & $19.66 \pm 5.27^{*}$ & 0.55 \\
Serum bicarbonate, venous (mmol/L) & $20.8(3.6)$ & $23.7(5.13)$ & 0.298 \\
Urea (mg/100ml) & $62.5(69.5)$ & $50(41.5)$ & 0.107 \\
VAC therapy duration (day) & $34.87 \pm 14.78^{*}$ & $31.04 \pm 18.8^{*}$ & 0.60 \\
Length of the hospital stay (day) & $34.5(29.25)$ & $41(28)$ & 0.696 \\
Intensive care period (day) & $27.5(16.25)$ & $1(5.50)$ & 0.001 \\
\hline
\end{tabular}

Median (IQR)

${ }^{*}$ Mean \pm Standard Derivation, $t$-test

The results were evaluated using t-test for parametric values. For nonparametric values, the results were evaluated using the Mann-Whitney $U$ test, Chi-square, and receiver operating characteristic (ROC) tests in addition to a regression analysis. Differences at the level of $p \leq 0.05$ were accepted as statistically significant. These statistical analyses were performed by using SPSS software (version 23.0, IBM Corporation, Armonk,
Table III: The distribution and statistical significance of the scores between groups according to FGSI and UFGSI scoring systems.

\begin{tabular}{|c|c|c|c|}
\hline $\begin{array}{l}\text { Temperature score } \\
0 \\
1\end{array}$ & $\begin{array}{l}\text { Group } 1 \text { (Exitus) } \\
\qquad \begin{array}{l}3(\% 13.6) \\
5(\% 62.5)\end{array}\end{array}$ & $\begin{array}{l}\text { Group } 2 \text { (Alive) } \\
\qquad \begin{array}{c}19(\% 86.4) \\
3(\% 37.5)\end{array}\end{array}$ & $\begin{array}{c}p \\
0.007\end{array}$ \\
\hline $\begin{array}{l}\text { Heart rate score } \\
0 \\
2 \\
\end{array}$ & $\begin{array}{l}4(\% 16.7) \\
4(\% 66.7) \\
\end{array}$ & $\begin{array}{c}20(\% 83.3) \\
2(\% 33.3) \\
\end{array}$ & 0.013 \\
\hline $\begin{array}{l}\text { Respiratory rate score } \\
0 \\
1 \\
\end{array}$ & $\begin{array}{l}5(\% 22.2) \\
3(\% 66.7)\end{array}$ & $\begin{array}{c}21(\% 77.8) \\
1(\% 33.3) \\
\end{array}$ & 0.019 \\
\hline $\begin{array}{l}\text { Serum potassium score } \\
0 \\
1 \\
2 \\
\end{array}$ & $\begin{array}{c}1(\% 6.7) \\
5(\% 38.5) \\
2(\% 100)\end{array}$ & $\begin{array}{c}14(\% 93.3) \\
8(\% 61.5) \\
0(\% 0)\end{array}$ & 0.009 \\
\hline $\begin{array}{l}\text { Serum sodium score } \\
0 \\
2 \\
\end{array}$ & $\begin{array}{c}7(\% 26.9) \\
1(\% 25) \\
\end{array}$ & $\begin{array}{c}19(\% 73.1) \\
3(\% 75) \\
\end{array}$ & 0.93 \\
\hline $\begin{array}{l}\text { Serum creatinine score } \\
0 \\
2 \\
3 \\
4 \\
\end{array}$ & $\begin{array}{c}4(\% 25) \\
3(\% 25) \\
1(\% 100) \\
0(\% 0)\end{array}$ & $\begin{array}{c}12(\% 75) \\
9(\% 75) \\
0(\% 0) \\
1(\% 100) \\
\end{array}$ & 0.36 \\
\hline $\begin{array}{l}\text { Haematocrit score } \\
0 \\
2 \\
\end{array}$ & $\begin{array}{c}5(\% 20.8) \\
3(\% 50)\end{array}$ & $\begin{array}{c}19(\% 79.2) \\
3(\% 50)\end{array}$ & 0.148 \\
\hline $\begin{array}{l}\text { White blood score } \\
0 \\
1 \\
2\end{array}$ & $\begin{array}{c}1(\% 16.7) \\
3(\% 30) \\
4(\% 28.6)\end{array}$ & $\begin{array}{c}5(\% 83.3) \\
7(\% 70) \\
10(\% 71.4)\end{array}$ & 0.82 \\
\hline $\begin{array}{l}\text { Serum bicarbonate score } \\
0 \\
1 \\
2 \\
3 \\
4 \\
\end{array}$ & $\begin{array}{c}1(\% 6.3) \\
1(\% 50) \\
5(\% 50) \\
1(\% 100) \\
0(\% 0) \\
\end{array}$ & $\begin{array}{c}14(\% 93.8) \\
1(\% 50) \\
5(\% 50) \\
0(\% 0) \\
1(\% 100) \\
\end{array}$ & 0.068 \\
\hline $\begin{array}{l}\text { Dissemination score } \\
\text { Limited to urogenital or } \\
\text { anorectal region } \\
\text { Limited in the pelvis } \\
\text { Passing out pelvis }\end{array}$ & $\begin{array}{c}3(\% 14.3) \\
1(\% 100) \\
4(\% 50)\end{array}$ & $\begin{array}{c}18(\% 85.7) \\
0(\% 0) \\
4(\% 50) \\
\end{array}$ & 0.036 \\
\hline $\begin{array}{l}\text { Age score } \\
\geq 60 \text { years } \\
<60 \text { years }\end{array}$ & $\begin{array}{l}6(\% 46.2) \\
2(\% 11.8)\end{array}$ & $\begin{array}{c}7(\% 53.8) \\
15(\% 88.2)\end{array}$ & 0.035 \\
\hline
\end{tabular}

NY, USA). Shapiro-Wilks, detrended plots, skewness/ kurtosis, coefficient of variation tests were used for normal distribution research. Nonparametric tests were used because these selected groups were not in normal distribution. Details of descriptive statistics were shown in Tables I-III.

\section{RESULTS}

Of the 30 patients included in the present study, 16 $(53.3 \%)$ were males and $14(46.7 \%)$ were females. The mortality rate observed in this study was $26.6 \%(n=8)$. No statistically significant sex-based difference was found between Group 1 and Group 2 (Table I). The mean age of the total patient population was $58.7 \pm 11.5$ years. 
Table IV: FGSI and UFGSI scoring systems for predicting mortality in patients with Fournier's gangrene.

\begin{tabular}{|c|c|c|c|c|c|c|}
\hline & Cut off $95 \% \mathrm{Cl}$ & Sensitivity & Specifity & Likelihood ratio & (+) Predictive value & (-) Predictive value \\
\hline UFGSI & $\geq 10$ & $87.5 \%$ & $96 \%$ & $29.25 \%$ & $80 \%$ & $100 \%$ \\
\hline FGSI & $\geq 7$ & $87.5 \%$ & $91 \%$ & $22.79 \%$ & $83.3 \%$ & $87.5 \%$ \\
\hline
\end{tabular}

FGSI: Fournier's Gangrene Severity Index; UFGSI: Uludag Fournier's Gangrene Severity Index.

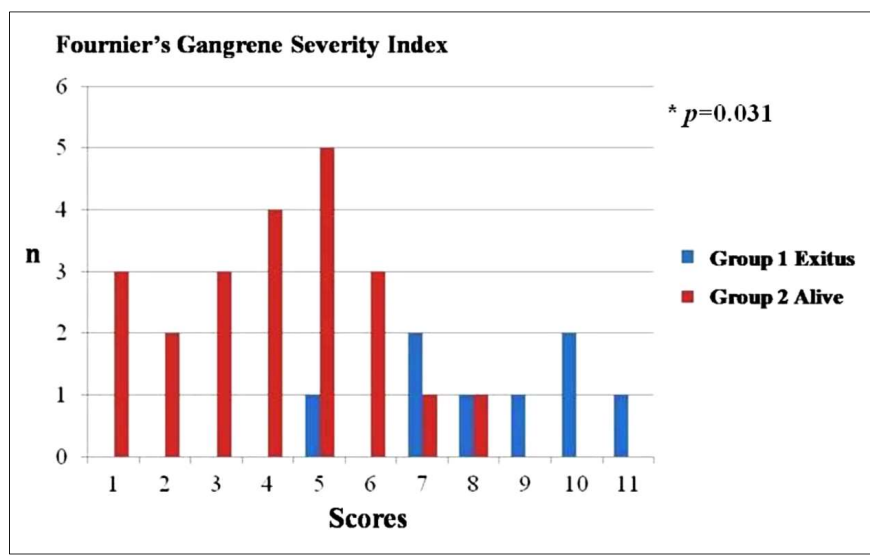

Figure 1: The distribution of patients according to the Fournier's Gangrene Severity Index scoring system.

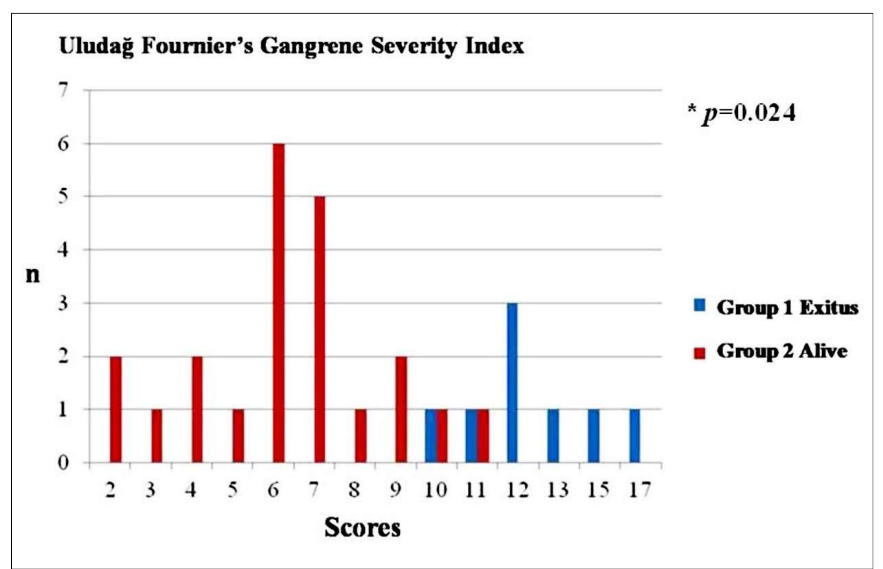

Figure 2: The distribution of patients according to the Uludag Fournier's Gangrene Severity Index scoring system.

The average age in Group 1 (68.5 \pm 12.08 years) was significantly higher than in Group 2 (55.13 \pm 9.17 years, $p=0.017$, Table II). A significant difference between the two groups regarding the age score of the UFGSI parameters was found $(p=0.035$, Table III). The dissemination score of infection was one of the UFGSI parameters significantly increased in Group $1(p=0.036)$ (Table III). According to UFGSI score, the scores of Group 1 and 2 patients significantly increased in Group 1 $(p=0.024)$. Similarly, according to the FGSI score, the scores of Group 1 and 2 patients significantly increased in Group 1 ( $p=0.031)$. According to Group 2, body temperature, heart and respiratory rate scores, serum potassium and hematocrit values (UFGSI and FGSI parameters) were significantly increased in Group 1 (Table II). In terms of Group 2, the heart and respiratory rate scores (UFGSI and FGSI scores) significantly increased in Group 1 ( $p=0.013$ and $p=0.019$, Table III).

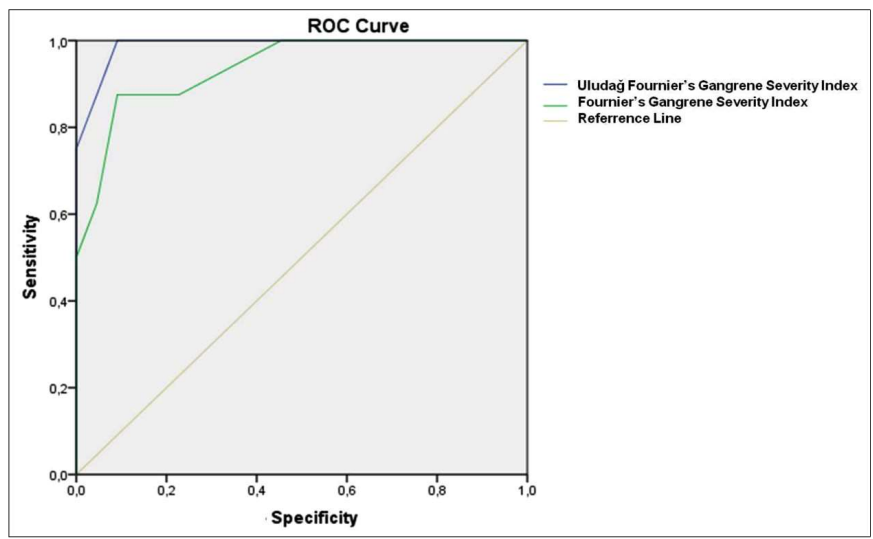

Figure 3: The predictive values of Uludag Fournier's Gangrene Severity Index and Fournier's Gangrene Severity Index scoring systems were evaluated by using receiver operating characteristic (ROC) curve analysis.

Group 1 and 2 contain seven and 21 patients with diabetes mellitus (DM), respectively. No significant difference was found between the incidence of DM in Group 1 and Group 2 (Table I). Three patients with DM of Group 1 and eight patients with DM of Group 2 were obese, too. A significant difference between the groups about obesity was not seen (Table I). In Group 1 eight patients and in Group 2 twelve patients had comorbidities aside from DM or obesity and a significant difference between the groups was seen $(p=0.02$, Table I). These 20 patients required mechanical ventilation support during intensive care unit due to respiratory failure and eight of these patients $(40 \%)$ died. In addition, ICP significantly increased in Group 1 according to Group 2 ( $p=0.001$, Table II).

Bacteria were isolated from wounds of FG in 21 patients. Bacteria were identified with deep tissue biopsy culture in 6 patients of Group 1 and in 15 patients of Group 2. Escherichia coli, isolated from 10 patients, was the most common of these infection-causing bacteria. Other bacteria, such as Acinetobacter, Streptococcus, Staphylococcus aureus, Pseudomonas, and Klebsiella were identified in 11 patients. A significant difference between the groups regarding bacterial isolation was not seen (Table I).

To predict mortality, the UFGSI and FGSI had $87.5 \%$ sensitivity and $96 \%$ and $91 \%$ specificity, respectively. To predict mortality (sensitivity, specificity, likelihood ratio, positive predictive value, and negative predictive value) in the UFGSI and FGSI scoring systems, ROC graph that was drawn using the cut-off valuations are presented in Figure 3 and Table IV.

\section{DISCUSSION}

Although FG management is well described, there are issues that need to be clarified regarding mortality. Many factors were reported to influence mortality in previous 
studies on FG. In some previous studies, the female sex was advocated as a risk factor about increasing mortality. 8,10 In this study, six of female patients and two of the male patients died. It was seen that mortality increased in female patients (Table I). Consistent with the studies reported by Yilmazlar et al., gender is not an effective factor in mortality. 7,11 Age is an important subject that is always debated whether an effective factor in mortality.5,12,13 Despite the study reported by Marin et al., an association was found between age and mortality consistent with the study reported by Yilmazlar et al.7,12 This result is one of the most remarkable findings of this study.

The act of comorbid conditions on mortality was investigated in many other studies. Some of the comorbid conditions were asserted to have an important role in mortality.5,12 DM, discussed as an effective role on FG, was determined in 28 patients of this study. In mortal patients, seven had DM in Group 1 and 21 had DM in Group 2 (Table I). As described previously in other studies, even though the frequency of DM increased in the living group, no significant difference was found between DM and mortality in this study. 5,7,12 More than one comorbid illness such as malignancy, heart failure, and respiratory failure significantly affected mortality $(p=0.02)$ (Table I), but one comorbid disease did not affect mortality. This result is another of the remarkable findings of this study. As described, a malignancy was indicated as an independent risk factor among these comorbid conditions for mortality. 10 In these patients, three patients were diagnosed as having a malignancy in Group I. Five patients of Group 1 had other comorbid conditions such as heart and respiratory failure, in addition to DM or obesity. Using FGSI and UFGSI to predict mortality in patients with $F G$ have been compared in different studies that evaluated their strength. 5,12 Both FGSI and UFGSI were indicated to correlate to mortality in this study. In the study, $87.5 \%$ sensitivity was obtained in both systems. The specificity was $96 \%$ for UFGSI and $91 \%$ for FGSI. Yilmazlar et al. described the UFGSI scoring system and informed sensitivity of $94 \%$ and specificity of $81 \% .{ }^{7}$ The results in the study reported here are compatible with the study described by Yilmazlar et al. ${ }^{7}$ Roghmann et al. found these figures to be a sensitivity of $85 \%$ and a specificity of $67 \%$ for UFGSI. 5 The sensitivity and specificity of FGSI in the two studies mentioned were $88 \%-67 \%$ and $65 \%-100 \%$, respectively. 5,7 Czymek et al. described a sensitivity of $87 \%$ and a specificity of $77 \%$ for FGSI, while Laor et al. noted a sensitivity of $75 \%$ and specificity of $78 \% .6,8$ In the other study reported by Yilmazlar et al., no living patient was described in the patients with UFGSI scores of $\geq$ nine in a series including 120 cases. ${ }^{11}$ The total mortality rate reported in this study was $20.8 \%$. The cut-off values for UFGSI and FGSI were reported to be nine and seven, respectively. ${ }^{11} \mathrm{We}$ indicated that the cut-off values for UFGSI and FGSI were 10 and seven, respectively (Table IV). To be 10 of the cut-off value for UFGSI is another one of the most remarkable results of this study. In the study, eight of 12 cases with a score $>$ nine for UFGSI died (Figure 1) and seven of nine cases with a score of $>$ seven for FGSI died (Figure 2). We considered that a prompt debridement and effective treatment were performed in cases with high mortality rates.

Of the collective parameters for both FGSI and UFGSI, heart and respiratory rate scores varied significantly between the two groups $(p=0.013$ and $p=0.019$, respectively, Table III). Temperature, heart rate, respiratory rate, serum potassium, and hematocrit levels were also significantly different between the groups (Table II). Other studies have associated serum creatinine, hematocrit, and potassium levels with increased mortality. ${ }^{12,14}$ Roghmann et al. also describe a relationship between mortality and creatinine and hematocrit levels. ${ }^{5}$

Disease dissemination significantly increased in Group 1 relative to Group 2. There was an association between the dissemination of disease and mortality, which is consistent with the studies by Yilmazlar et al.7,11 Moreover, Roghmann et al. suggest using disease dissemination as a scoring system to predict prognosis and patient outcome. 5

In this study, HT was not significantly different between the two groups, whereas ICP significantly increased in Group 1 relative to Group 2 (Table II). Furthermore, ICP was associated with mortality; this represents another one of the most remarkable results from the present study. The mortality rate of the patients receiving mechanical ventilation assistance was $40 \%$ in this study and $68 \%$ in the study described by Yilmazlar et al. involving 120 patients. 11

In this study, bacteria were isolated from $75 \%$ of mortal patients. The most commonly found bacteria, E. coli was isolated from wounds of $F G$ in $33.3 \%$ of all patients. The type of bacterial reproduction was found similar to reported in the literature.5,15 Bacterial reproduction was not found to significantly associate with mortality (Table I).

In this study, the most frequently origin of FG was urogenital disease (56.6\%), followed by anorectal disease $(43.4 \%$, Table I). FG should be managed by surgeons or urologists because it is generally impossible to define the complete source of FG. The impact of infection source on mortality was not found to be statistically significant.

In the present study, a faecal diversion procedure containing sigmoid loop colostomy was performed in five patients (16.6\%) in Group 2 (Table I). We routinely perform enemas before changing VAC dressings every three to four days in the practice. We never recommend faecal diversion unless there is extensive sphincter damage or large perineal wounds. ${ }^{16}$ Thanks to VAC therapy, debridement regions are kept clean and heal quickly; furthermore, the number of debridements has decreased. Fecal diversion was not found to be a statistically significant risk factor for mortality. 
The most limiting aspect of this study was its retrospective design. Additionally, the number of patients enrolled in the present study is also low. Nevertheless, this study has rigid inclusion standards that ensure the uniformity of the patient population. In fact, an extensive series of $F G$ has been presented very little until today. The factors affecting mortality were analyzed in patients receiving the same treatment strategies for FG; therefore, the uniformity of the patient population and the similarity in treatment approach represents the greatest strength of this study.

\section{CONCLUSION}

Age and dissemination scores of disease were important prognostic indicators about the factors causing mortality in FG. Although many scoring systems have been used to determine mortality, we instead considered the age and dissemination scores of UFGSI. The issues concerning the study's hypothesis were investigated and all of our predictions (high mortality expectancy in patients with prolonged ICP, comorbidities, and high FGSI and UFGSI scores) were confirmed. It was found that being aged over 60 years and having the disease exceeding the pelvis are relevant parameters for predicting mortality. Patients with UFGSI score under 10 have high probabilities of survival and seldom require intensive care. Patients with UFGSI score over 10 have a higher risk of mortality. This latter group of patients should be under the direct care of an experienced clinical team including general surgeons, plastic surgeons, and intensive care specialists in an intensive care unit.

\section{CONFLICT OF INTEREST:}

Authors declared no conflict of interest.

\section{AUTHORS' CONTRIBUTION:}

SE, SK, SH: Contributed to the design of the work; analysis of data for the work; drafting the work, final approval of the manuscript, and agreement to be accountable for all aspects of the work.

BG, MY: Contributed to the design of the work; analysis, of data for the work; drafting the work.

\section{REFERENCES}

1. Morpurgo E, Galandiuk S. Fournier's gangrene. Surg Clin North Am 2002; 82:1213-24.
2. Sroczynski M, Sebastian M, Rudnicki J, Sebastian A, Agrawal AK. A complex approach to the treatment of Fournier's gangrene. Adv Clin Exp Med 2013; 22:131-5.

3. Thwaini A, Khan A, Malik A, Cherian J, Barua J, Shergill I, et al. Fournier's gangrene and its emergency management. Postgrad Med J 2006; 82:516-9.

4. Corcoran AT, Smaldone MC, Gibbons EP, Walsh TJ, Davies BJ. Validation of the Fournier's gangrene severity index in a large contemporary series. J Urol 2008; 180:944-8.

5. Roghmann F, Bodman C, Löppenberg B, Hinkel A, Palisaar J, Noldus $\mathrm{J}$. Is there a need for the Fournier' s gangrene severity index? Comparison of scoring systems for outcome prediction in patients with Fournier's gangrene. BJU Int 2012; 110:1359-65.

6. Hahn HM, Jeong KS, Park DH, Park MC, Lee IJ. Analysis of prognostic factors affecting poor outcomes in 41 cases of Fournier gangrene. Ann Surg Treat Res 2018; 95:324-32.

7. Yilmazlar T, Ozturk E, Ozguc H, Ercan I, Vuruskan H, Oktay B. Fournier's gangrene: An analysis of 80 patients and a novel scoring system. Tech Coloproctol 2010; 14:217-23.

8. Czymek R, Frank P, Limmer S, Schmidt A, Jungbluth T, Roblick U, et al. Fournier's gangrene: Is the female gender a risk factor? Langenbecks Arch Surg 2010; 395:173-80.

9. Ozturk E, Ozguc H, Yilmazlar T. The use of vacuum assisted closure therapy in the management of Fournier's gangrene. Am J Surg 2009; 197:660-5.

10. Taviloglu K, Cabioglu N, Cagatay A, Yanar H, Ertekin C, Baspinar I, et al. Idiopathic necrotizing fasciitis: Risk factors and strategies for management. Am Surg 2005; 71:315-20.

11. Yilmazlar T, Isik Ö, Öztürk E, Özer A, Gülcü B, Ercan I. Fournier's gangrene: Review of 120 patients and predictors of mortality. Ulus Travma Acil Cerrahi Derg 2014; 20:333-7.

12. García Marín A, Turégano Fuentes F, Cuadrado Ayuso M, Andueza Lillo JA, Cano Ballesteros JC, Pérez López M. Predictive factors for mortality in Fournier's gangrene: A series of 59 cases. Cir Esp 2015; 93:12-7.

13. Ulug M, Gedik E, Girgin S, Celen MK, Ayaz C. The evaluation of microbiology and Fournier's gangrene severity index in 27 patients. Int J Infect Dis 2009; 13:e424-30.

14. Lin TY, Ou CH, Tzai TS, Tong YC, Chang CC, Cheng HL, et al. Validation and simplification of Fournier's gangrene severity index. Int J Urol 2014; 21:696-701.

15. Wróblewska M, Kuzaka B, Borkowski T, Kuzaka P, Kawecki D, Radziszewski P. Fournier's gangrene - current concepts. Pol J Microbiol 2014; 63:267-73.

16. Ozturk E, Sonmez Y, Yilmazlar T. What are the indications for a stoma in Fournier's gangrene? Colorectal Dis 2011; 13:1044-7. 